\title{
The COVID-19 pandemic and pediatric mental health: advocating for improved access and recognition
}

\author{
Abdallah Dalabih $\mathbb{1 D}^{1 凶}$, Erin Bennett ${ }^{1}$, Joyce R. Javier ${ }^{2}$ and On behalf of the Pediatric Policy Council* \\ (c) The Author(s), under exclusive licence to the International Pediatric Research Foundation, Inc 2022
}

Pediatric mental health has been poorly addressed and access to quality psychiatric care is limited in many countries around the world including wealthy nations. The novel coronavirus disease 2019 (COVID-19) pandemic caused a strain on pediatric mental health resources across the globe. This was primarily due to the stress of lockdowns, loss of caregivers, and school interruptions, which further exacerbated the mental health needs of children. Despite their unreadiness, emergency departments have been utilized to address those needs. Kostopoulou et al. reported that, although emergency departments' pediatric visits have decreased earlier during the pandemic, mental health visits increased during the same period.

Pediatric Research (2022) 91:1018-1020; https://doi.org/10.1038/s41390-022-01952-w

Advocating for, and funding better access to specialized mental health care, building capacity through mental health training for primary care pediatricians, and school and community-based recognition and prevention programs should be a public health priority.

Pediatric mental health surfaced as prominent public health concern since the early days of the novel coronavirus disease 2019 (COVID-19) pandemic in 2020. In this issue, Kostopoulou et al. presented data from Greece showing a significant decline in general pediatric emergency department (ED) visits and increased hospitalization rates during the novel COVID-19 pandemic. The authors reported prospective data from one medical center in the first year of the pandemic. These data suggest that, although pediatric patients presented to the ED less often during the pandemic, they often had more severe symptoms when they did go to the ED. This observation may be related to delay care due to any lockdowns or families' fear of exposure to the virus. Despite the decrease in ED visits, the authors reported an increase in psychiatric ED visits and psychiatric admissions during the study period.

During the COVID-19 pandemic, a sharp decrease in pediatric ED visits has been reported by other authors. In a study from the United States by Pines et al., pediatric ED visits decreased by $74 \%$ through the week of April 15, 2020, compared to a $60 \%$ decline in adult visits. In that study, a recovery rate of $72 \%$ occurred for children aged $14-17$ years by June. However, only a $50 \%$ recovery rate was observed for children aged $<10$ years. This decline was seen across all ED types and locations and across all diagnoses. ${ }^{1}$ This trend was observed by other authors in different countries and settings; a study from Canada showed similar findings for pediatric ED visits across the country, and a Dutch group showed underutilization of pediatric ED services during the lockdown in the Netherlands. ${ }^{2,3}$

The increases in psychiatric ED visits and psychiatric admissions during the study period are consistent with other reports from around the globe showing a correlation between the pandemic and an increase in the diagnosis and reporting of mental health disorders. The Centers for Disease Control and Prevention reported a 24\% (ages 5-11) and 31\% (ages 12-17) increase in ED visits for mental health emergencies after April 2020 compared to $2019 .{ }^{4}$ This was hypothesized to be related to the disruptions caused by the lockdowns and the sense of isolation and uncertainty. In addition, globally, over 1 million children have lost primary caregivers to COVID-19, and there have been increases in reports of domestic violence in households. ${ }^{5-7}$

The Centers for Disease Control and Prevention (CDC) reported a $33 \%$ increase in mental health diagnoses in children in the first two quarters of 2021, a $103 \%$ increase in suicide rates compared to 2016 , and a $50 \%$ increase in suicide attempts in girls aged 12-17 years in early 2021 compared to $2019 .^{8}$ Hill et al. reported an increase in the rates of suicidal ideation and attempts during 2020 compared with 2019. Although those were not universally higher across that period, they found that periods with significantly higher rates of suicide-related behaviors corresponded to times when COVID-19-related stressors and community responses were heightened. ${ }^{9}$ This high rate of suicidal behavior and mental health diagnoses coupled with inadequate primary care providers' readiness to address them will likely have a profound effect on children's wellbeing for years to come. Addressing the mental health crisis is particularly critical for communities of color in the US who have been disproportionately affected by COVID-19 infections, COVID-related caregiver loss, and racism and discrimination and often face increased mental health stigma. $7,10-12$

This rise in patients presenting to EDs with mental health disorders, as well as their acuity, will be challenging to many EDs. The 2019 National Pediatric Readiness Assessment (NPRP) collaborative, which was founded to ensure that all US EDs have the guidelines and resources to provide effective emergency care

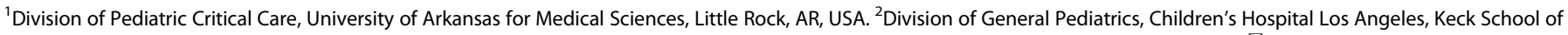
Medicine, University of Southern California, Los Angeles, CA, USA. *A list of authors and their affiliations appears at the end of the paper. ${ }^{\circledR}$ email: Adalabih@uams.edu 
to children, found that only $47.2 \%$ of EDs had children's mental health policy. It was even lower in rural areas (33\%). The NPRP has also found that children treated in EDs that do not see children often had worse outcomes, including increased mortality. ${ }^{13}$ For this reason, a new approach to address this growing crisis should be developed. Public mental health interventions should be part of a comprehensive public health emergency response plan worldwide. This approach should focus on building capacity through community programs-both outpatient and inpatienthopefully decreasing the need to seek acute mental health care in ED settings.

Access to high-quality mental health services is an even more complicated problem in low- and middle-income countries (LMIC). A study by Wainberg et al. identified four priority areas to diminish the mental health treatment gap and to improve access to highquality mental health services globally: (1) diminishing mental health stigma, (2) building treatment and research capacity, (3) implementing prevention programs, and (4) establishing a sustainable scale-up of public health systems to improve access to mental health treatment using evidence-based interventions. ${ }^{14}$ In the United States, some programs have been developed to fund initiatives aiming to increase access to high-quality mental health services. For example, the American Rescue Plan Act-Pediatric Mental Health Care Access Program aims to make early identification, diagnosis, treatment, and referral of behavioral disorders a routine part of children's health care services. The program has funded close to 50 state and regional networks of pediatric mental health care teams in 40 states to achieve this. Those teams would provide behavioral health services through telehealth to pediatric primary care providers. Continuing funding of this program and expanding it to states and regions where it is not currently utilized will help develop the workforce capacity to address the urgent need for mental health services.

In October 2021, the American Academy of Pediatrics, the American Academy of Child and Adolescent Psychiatry, and the Children's Hospital Association joined together to declare the National State of Emergency in Children's Mental Health. It proposed 10 ways policymakers can address this emergency. These strategies include advocating for an equitable increase in federal funding from infancy through adolescence so that all families and children can access evidence-based mental health screening, diagnosis, and treatment, improving access to telemedicine to provide mental health care to all populations, focusing on sustainable funding of school-based mental health care, strengthening suicide prevention efforts, and accelerating strategies to workforce challenges in child mental health. ${ }^{15}$

In the United States, specific legislation has been proposed in response to this national state of emergency. For instance, The Mental Health Services for Students Act aims to provide funding for public schools to partner with local mental health professionals to establish on-site mental health services for students. With this act, access to mental health services will improve, and thousands of children can be assisted. In the limited areas where it is implemented, this approach has proven to be successful in helping students overcome mental health issues and improving the quality of life for themselves and their families. The Mental Health Services for Students Act would expand this model across the United States to provide more students on-site mental health care. ${ }^{16}$ Other countries have recognized that pediatric mental health care access may be addressed by bringing the care to the child at school. A report by the Association of Southeast Asian Nations found that school-based mental health varied between the member countries, and only Singapore conducted mental health training for teachers in a comprehensive way. ${ }^{17}$

In summary, the COVID-19 pandemic has exposed and deepened the pediatric mental health crisis globally. The stress of the lockdown, school closures, loss of caregivers, and political tensions are actual, and children need professional mental health support. The ED is not the ideal location for providing this service, and improving access to intervention and preventive mental health services through the utilization of primary care providers and school-based, and community-based services (i.e., churches, community-based organizations) may be of value to those of us who are struggling to provide appropriate support for children and their caregivers. ${ }^{18-20}$

\section{REFERENCES}

1. Pines, J. M. et al. Characterizing pediatric emergency department visits during the COVID-19 pandemic. Am. J. Emerg. Med. 41, 201-204 (2021).

2. Kruizinga, M. D. et al. The impact of lockdown on pediatric ED visits and hospital admissions during the COVID19 pandemic: a multicenter analysis and review of the literature. Eur. J. Pediatr. 180, 2271-2279 (2021).

3. Finkelstein, Y. et al. Effect of the COVID-19 pandemic on patient volumes, acuity, and outcomes in pediatric emergency departments: a nationwide study. Pediatr. Emerg. Care. 37, 427-34. (2021).

4. Leeb R. T. et al. Mental health-related emergency department visits among children aged $<18$ years during the COVID-19 pandemic - United States, January 1-October 17, 2020. MMWR Morb. Mortal Wkly Rep. 69, 1675-1680 (2020).

5. Kumar, A. \& Nayar, K. R. COVID 19 and its mental health consequences. J. Ment. Health 30, 1-2 (2021).

6. Bhattacharjee, B. \& Acharya, T. The COVID-19 pandemic and its effect on mental health in USA - a review with some coping strategies. Psychiatr. Q. 91, 1135-45. (2020).

7. Hillis, S. D. et al. Global minimum estimates of children affected by COVID-19associated orphanhood and deaths of caregivers: a modelling study. Lancet $\mathbf{3 9 8}$, 391-402 (2021).

8. Yard, E. et al. Emergency department visits for suspected suicide attempts among persons aged 12-25 years before and during the COVID-19 pandemic - United States, January 2019-May 2021. MMWR Morb. Mortal. Wkly Rep. 70, 888-894 (2021).

9. Hill, R. M. et al. Suicide ideation and attempts in a pediatric emergency department before and during COVID-19. Pediatrics 147, e2020029280 (2021).

10. Nau, C. et al. COVID-19 inequities across multiple racial and ethnic groups: results from an integrated health care organization. Ann. Intern. Med. 174, 1183-1186 (2021).

11. Dreyer, B. P. et al. The death of George Floyd: bending the arc of history toward justice for generations of children. Pediatrics 146, e2020009639 (2020).

12. Beers, L. S. et al. Immunizing against hate: overcoming Asian American and Pacific Islander racism. Pediatrics 148, e2021051836 (2021).

13. Remick, K. et al. Pediatric readiness in the emergency department. Ann. Emerg. Med. 72, e123-e136 (2018).

14. Wainberg, M. L. et al. Challenges and opportunities in global mental health: a research-to-practice perspective. Curr. Psychiatry Rep. 19, 28 (2017).

15. AAP-AACAP-CHA. AAP-AACAP-CHA Declaration of a National Emergency in Child and Adolescent Mental Health. https://www.aap.org/en/advocacy/child-andadolescent-healthy-mental-development/aap-aacap-cha-declaration-of-anational-emergency-in-child-and-adolescent-mental-health/ (2021).

16. 117th Congress H.R. 721, Mental Health Services for Students Act of 2021, Bill Profile (2021)

17. Nishio, A., Kakimoto, M., Bermardo, T. M. S. \& Kobayashi, J. Current situation and comparison of school mental health in ASEAN countries. Pediatr. Int. 62, 438-443 (2020).

18. Javier, J. R. et al. Promoting enrollment in parenting programs among a Filipino population: a randomized trial. Pediatrics 143, e20180553 (2019).

19. Javier, J. R., Coffey, D. M., Schrager, S. M., Palinkas, L. A. \& Miranda, J. Parenting intervention for prevention of behavioral problems in elementary school-age Filipino-American children: a pilot study in churches. J. Dev. Behav. Pediatr. 37, 737-745 (2016).

20. Javier, J. R. et al. Recruiting Filipino immigrants in a randomized controlled trial promoting enrollment in an evidence-based parenting intervention. J. Immigr. Minor Health 21, 324-331 (2019).

\section{AUTHOR CONTRIBUTIONS}

Abdallah Dalabih: manuscript conception and design, drafting and critically revising, and providing final approval for publication. Erin Bennett: provided critical revisions and final approval of the manuscript. Joyce Javier: Manuscript design, critical revisions and final approval for publication. 


\section{COMPETING INTERESTS}

The authors declare no competing interests.

\section{ETHICS APPROVAL AND CONSENT TO PARTICIPATE}

No patient data were needed for this manuscript, and no patient consents were required.

\section{ADDITIONAL INFORMATION}

Correspondence and requests for materials should be addressed to Abdallah Dalabih.

Reprints and permission information is available at http://www.nature.com/ reprints

Publisher's note Springer Nature remains neutral with regard to jurisdictional claims in published maps and institutional affiliations.

\section{ON BEHALF OF THE PEDIATRIC POLICY COUNCIL}

Shetal Shah ${ }^{3}$, Jean Raphael ${ }^{4}$, Mona Patel ${ }^{4}$, Jonathan Davis ${ }^{5}$, DeWayne Pursley ${ }^{5}$, Tina Cheng ${ }^{6}$, Sherin Devaskar ${ }^{6}$, Joyce Javier ${ }^{7}$ and Lois Lee ${ }^{7}$

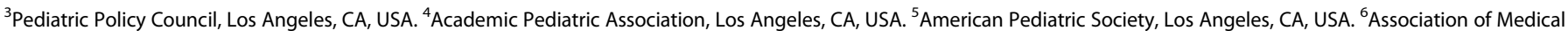
School Pediatric Department Chairs, Los Angeles, CA, USA. ${ }^{7}$ Society for Pediatric Research, Los Angeles, CA, USA. 